\title{
WEAK MAJORIZATION INEQUALITIES FOR SINGULAR VALUES
}

\section{Limin ZOU AND ChUANJiAng HE}

Abstract. In this paper, we refine an inequality due to Bhatia and Kittaneh [Linear Algebra Appl. 308 (2000) 203-211], and generalize another inequality by Bhatia and Kittaneh [Lett. Math. Phys. 43 (1998) 225-231].

Mathematics subject classification (2010): 15A18, 15A42, 47A63.

Keywords and phrases: Weak majorization, singular values, positive semidefinite matrices.

\section{REFERENCES}

[1] R. Bhatia, Matrix Analysis, Springer-Verlag, New York, 1997.

[2] X. Zhan, Matrix Inequalities, Lecture Notes in Mathematics, vol. 1790, Springer-Verlag, Berlin, 2002.

[3] F. Zhang, Matrix Theory-Basic Results and Techniques, Springer-Verlag, New York, 2011.

[4] R. Bhatia, F. Kittaneh, Notes on matrix arithmetic-geometric mean inequalities, Linear Algebra Appl. 308 (2000), 203-211.

[5] X. ZHAN, Singular values of differences of positive semidefinite matrices, SIAM J. Matrix Anal.Appl. 22 (2000), 819-823.

[6] R. Bhatia, F. Kittaneh, Norm inequalities for positive operators, Lett.Math.Phys. 43 (1998), 225 231.

[7] R. Bhatia, F. Kittaneh, On the singular values of a product of operators, SIAM J.Matrix Anal.Appl. 11 (1990), 272-277.

[8] F. HIAI, H. KoSAKI, Means for matrices and comparison of their norms, Indiana Univ.Math.J. 48 (1999), 899-936.

[9] J.-C. Bourin, A matrix subadditivity inequality for symmetric norms, Proc.Amer.Math.Soc. 138 (2010), 495-504.

[10] R. Bhatia, F. KitTAneh, The matrix arithmetic-geometric mean inequality revisited, Linear Algebra Appl 428 (2008), 2177-2191.

[11] R. Bhatia, Positive Definite Matrices, Princeton University Press, Princeton, 2007.

[12] S. W. DruRY, On a question of Bhatia and Kittaneh, Linear Algebra Appl. 437 (2012), 1955-1960.

[13] Y. TAO, More results on singular value inequalities of matrices, Linear Algebra Appl. 416 (2006), 724-729. 